\title{
Analysis of GAGE, NY-ESO-1 and SP17 cancer/testis antigen expression in early stage non-small cell lung carcinoma
}

\author{
Morten F Gjerstorff ${ }^{*}$, Mette Pøh ${ }^{2,3}$, Karen E Olsen ${ }^{2,4}$ and Henrik J Ditzel ${ }^{1,3}$
}

\begin{abstract}
Background: The unique expression pattern and immunogenic properties of cancer/testis antigens make them ideal targets for immunotherapy of cancer. The MAGE-A3 cancer/testis antigen is frequently expressed in non-small cell lung cancer (NSCLC) and vaccination with MAGE-A3 in patients with MAGE-A3-positive NSCLC has shown promising results. However, little is known about the expression of other cancer/testis antigens in NSCLC. In the present study the expression of cancer/testis antigens GAGE, NY-ESO-1 and SP17 was investigated in patients with completely resected, early stage, primary NSCLC.

Methods: Tumor biopsies from normal lung tissue and from a large cohort $(n=169)$ of NSCLC patients were examined for GAGE, NY-ESO-1 and SP17 protein expression by immunohistochemical analysis. The expression of these antigens was further matched to clinical and pathological features using univariate cox regression analysis.

Results: GAGE and NY-ESO-1 cancer/testis antigens were not expressed in normal lung tissue, while SP17 was expressed in ciliated lung epithelia. The frequency of GAGE, NY-ESO-1 and SP17 expression in NSCLC tumors were $26.0 \%$ (44/169), 11.8\% (20/169) and 4.7\% (8/169), respectively, and 33.1\% (56/169) of the tumors expressed at least one of these antigens. In general, the expression of GAGE, NY-ESO-1 and SP17 was not significantly associated with a specific histotype (adenocarcinoma vs. squamous cell carcinoma), but high-level GAGE expression (>50\%) was more frequent in squamous cell carcinoma $(p=0.02)$. Furthermore, the frequency of GAGE expression was demonstrated to be significantly higher in stage II-IIla than stage I NSCLC (17.0\% vs. 35.8\%; $p=0.02)$. Analysis of the relation between tumor expression of GAGE and NY-ESO-1 and survival endpoints revealed no significant associations.

Conclusion: Our study demonstrates that GAGE, NY-ESO-1 and SP17 cancer/testis antigens are candidate targets for immunotherapy of NSCLC and further suggest that multi-antigen vaccines may be beneficial.
\end{abstract}

Keywords: Cancer/testis antigen, Immunotherapy, GAGE, NY-ESO-1, SP17, Lung cancer

\section{Background}

Harnessing the power of our immune system has long been a promising approach to treating cancer, and a large number of tumor antigens that might be used as targets for immunotherapy have been identified. Cancer/testis (CT) antigens are among the most promising due to their highly restricted expression to immune privileged cells of the testis and placenta in normal tissues as well as their natural immunogenic properties [1,2]. Current strategies

\footnotetext{
* Correspondence: mgjerstorff@health.sdu.dk

'Department of Cancer and Inflammation Research, Institute for Molecular Medicine (IMM), University of Southern Denmark, Winsloewparken 25, 3, DK-5000 Odense C, Denmark

Full list of author information is available at the end of the article
}

employing CT antigens as targets for immunotherapy include vaccination $[1,3]$ and adoptive transfer of $\mathrm{T}$ cells with genetically-modified $\mathrm{T}$ cell receptors [4-6].

Non-small cell lung cancer (NSCLC) accounts for more than $85 \%$ of new cases of lung cancer, which is the leading cause of cancer deaths worldwide [7], highlighting the need for novel therapeutic strategies for treatment of this disease. Cellular and humoral immune responses to CT antigens have been reported in NSCLC patients [8-14], suggesting that these proteins may be candidate targets for cancer immunotherapy of NSCLC. In addition, CT antigen sero-reactivity may be of diagnostic value for NSCLC patients [15]. Interestingly, adjuvant therapy

\section{Biomed Central}


with a MAGE-A3 CT antigen vaccine in patients with MAGE-A3-positive NSCLC has shown promising results [16], and allogeneic lymphocytes expressing recombinant T-cell receptors recognizing CT antigens NY-ESO-1 and MAGE-A3 were recently shown to effectively kill lung cancer cells [4]. This suggests that cancer immunotherapy targeting CT antigens may be an effective treatment for NSCLC. However, characterization of additional targets in NSCLC is needed to further develop broadly applicable, effective and specific immunotherapy regimens.

An important factor to consider when selecting appropriate targets for cancer immunotherapy is the expression frequency within the cancer of interest. In this study, we report a systematic analysis of the expression of the CT antigens GAGE, NY-ESO-1 and SP17 in early-stage NSCLC. NY-ESO-1 and the GAGE multi-gene family are members of the chromosome X-encoded CT antigens, which generally exhibit complete testis-specificity and are expressed at the spermatogonial stage of spermatogenesis [2]. In contrast, autosomal encoded CT antigens, such as SP17, are characterized by low expression in a limited number of non-testis, normal, tissues and tend to be expressed in the late stages of spermatogenesis. Our results will enhance the selection of appropriate targets for immunotherapeutic treatment of this disease.

\section{Methods}

\section{Tumor samples}

NSCLC surgical resection specimens were collected as diagnostic specimens from patients treated at the University Hospital of Odense from 1992-1999. The experiment was conducted in compliance with the Helsinki declaration and was approved by the ethical committee of Funen and Vejle County (VF20050069). Informed consent from participants was not needed for this type of experiment. All patients had undergone complete surgical resection without further treatment (neoadjuvant or adjuvant chemo- or radiotherapy). The histological subtypes of the tumors were established by morphology using light microscopy or by TTF1 and p63 status using immunohistochemistry. Formalin-fixed and paraffin-embedded tumor sections were stained with hematoxylin and eosin, and two $1 \mathrm{~mm}$ cores were punched from the central part of the tumors were transferred to tissue microarrays for further analysis.

\section{Immunohistochemical staining}

Methods for immunohistochemical staining of GAGE, NY-ESO-1 and SP17 in formalin-fixed, paraffin-embedded tissues and the characteristics of the antibodies used have been described previously [17-19]. Notably, the anti-GAGE antibody likely recognizes all members of the GAGE family. In brief, tissue sections were cut, deparaffinized, treated with $1.5 \% \mathrm{H}_{2} \mathrm{O}_{2}$ in Tris-buffered saline $(\mathrm{pH} 7.5)$ for $10 \mathrm{~min}$ to block endogenous peroxidase activity, rinsed in distilled $\mathrm{H}_{2} \mathrm{O}$, demasked for antigen retrieval and washed in TNT buffer $(0.1 \mathrm{M}$ Tris, $0.15 \mathrm{M} \mathrm{NaCl}$, $0.05 \%$ Tween-20, pH 7.5). Primary monoclonal antibodies (anti-GAGE (clone M3) [18], 1:100; anti-NY-ESO-1 (clone E978) [18] 1:25; anti-SP17 (clone 22) [17] 1:400) were diluted in antibody diluent (DAKO Cytomation, Glostrup, Denmark) and added to sections for $1 \mathrm{~h}$ at room temperature. Sections were washed with TNT and incubated with horseradish peroxidase-conjugated Envision or Powervision polymer (DAKO Cytomation) for $30 \mathrm{~min}$, followed by another wash with TNT. The final reaction product was visualized by incubating with 3,3 '-diaminobenzidine $(\mathrm{DAB})$ + substrate-chromogen for $10 \mathrm{~min}$, followed by washing with $\mathrm{H}_{2} \mathrm{O}$ and counterstaining of sections with Mayers hematoxylin before mounting in AquaTex (Merck Inc., Whitehouse Station, NJ, USA).

\section{Histological evaluation}

Immunohistochemical staining was evaluated for percentage of positive tumor cells by a skilled pathologist. Since positively-stained cells were generally strongly stained, differences in intensity was not assessed. The specimens were scored in four categories: $0(\leq 1 \%), 1(>1 \%-\leq 10 \%)$, $2(>10 \%-\leq 50 \%)$ and $3(>50 \%)$. Cells were considered positive if staining was convincingly observed in either the cytoplasm or the nuclei, or both, regardless of intensity. The cores were reported as missing if none or few tumor cells were present $(<30$ cells).

\section{Statistical analysis}

Univariate regression analysis using Cox proportionalhazard models and Kaplan-Meier survival analysis was performed using STATA software. The comparison of $\mathrm{CT}$ antigen expression with histotype and clinical stage was analyzed with the two-sided chi-squared test using a 5\% significance level. Analysis of CT antigen co-expression was done with the $\mathrm{Z}$ test comparing expected and observed proportions of positive tumors.

\section{Results and discussion}

We evaluated the expression of GAGE, NY-ESO-1 and SP17 CT antigens in normal lung tissue $(\mathrm{n}=5)$ and tumors from 169 patients with completely resected, early stage primary NSCLC. Patient characteristics are presented in Table 1. GAGE, NY-ESO-1 and SP17 expression was examined using well-characterized antibodies and previously established methods for immunohistochemical staining $[17,18]$. GAGE and NY-ESO-1 was not detected in normal lung tissues, but SP17 was expressed in a subset of ciliated epithelial cells of the bronchi (Figure 1), in accordance with previously published data $[17,18]$. As shown in Table 2, GAGE proteins were detected in $26.0 \%$ $(44 / 169)$ of NSCLC tumors and in $63.6 \%(28 / 44)$ of the 
Table 1 Characteristics of patients included in the study

\begin{tabular}{llll}
\hline Pathological parameter & Cases & $\%$ \\
\hline Gender & Male & 104 & 62.1 \\
\multirow{3}{*}{ Histology } & Female & 65 & 37.9 \\
\cline { 2 - 4 } & Adenocarcinoma & 81 & 47.9 \\
& Squamous cell carcinoma & 84 & 49.7 \\
\hline TNM stage & NOS* & 4 & 2.4 \\
& IA & 31 & 18.3 \\
& IIA & 57 & 33.7 \\
& IIB & 40 & 23.7 \\
& IIIA & 17 & 10.1 \\
\end{tabular}

*NOS 'Not otherwise specified'. Negative for adenocarcinoma marker TFF1 and squamous cell carcinoma marker p63.

positive tumors there were more than $50 \%$ positive tumor cells. This demonstrates that the expression frequency of GAGE proteins in NSCLC is similar to that of MAGE-A3, which is currently being tested as a vaccine target in NSCLC [16,20], as mentioned above. All GAGE-positive tumor cells exhibited cytoplasmic staining, but there were clear differences in the level of nuclear staining among and within tumors, ranging from absent to intense (Figure 1). This subcellular distribution is in agreement with a previous report on GAGE protein expression in a small set of lung cancers and other types of cancer [18]. NY-ESO-1 was detected in 11.8\% (20/169) of tumors and, as with GAGE, the distribution in most tumors was near homogenous ( $>50 \%$ positive tumor cells in 14 of 20 tumors examined) (Table 2). This expression frequency is in line with previous studies reporting 8.3-25\% NY-ESO1-positive NSCLC tumors [20-23]. The discrepancy in reported frequencies of NY-ESO-1 expression may be due to multiple parameters such as variation in clinical material (sampling, stage, subtype, treatment), staining protocol, scoring system/personnel etc. It is possible that the present study, using two $1 \mathrm{~mm}$ cores per tumor, may have included more false negatives than studies using whole tumor sections. However, NY-ESO-1 was relatively homogenously expressed in the majority of NSCLC tumors analyzed, supporting the validity of the two-core approach. The subcellular localization of NY-ESO-1 in NSCLC tumors was predominantly cytoplasmic. SP17 was detected in $4.7 \%(8 / 169)$ NSCLC tumors, which is comparable to existing targets of NSCLC (e.g. EML4-ALK) $[24,25]$. In all 8 positive tumors, less than $10 \%$ of the tumor cells were positive. Notably, the SP17-positive tumor cells exhibited a scattered distribution within tumors in contrast to GAGE and NY-ESO-1, which were most often either

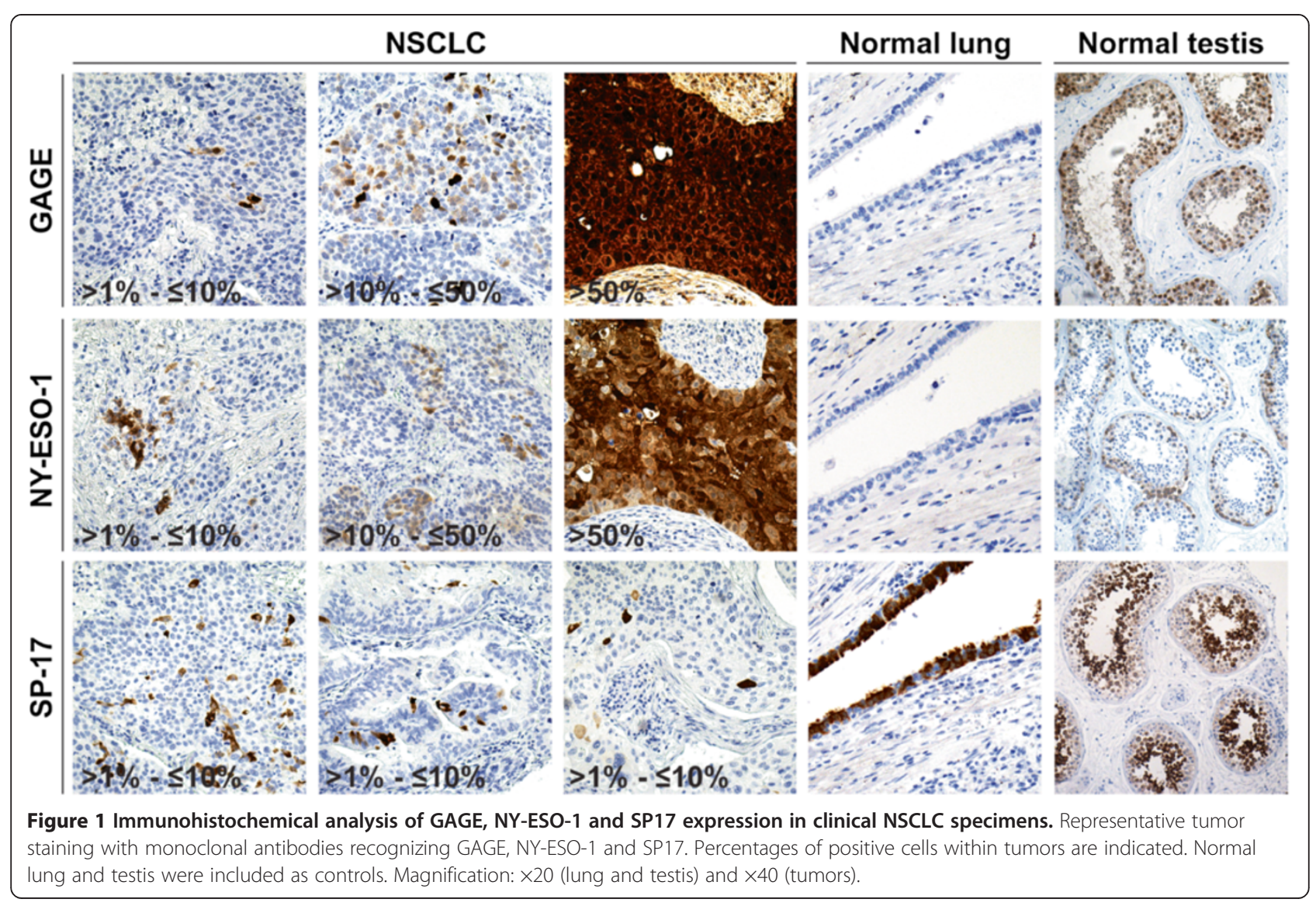


Table 2 Expression of GAGE, NY-ESO-1 and SP17 in human NSCLC

\begin{tabular}{|c|c|c|c|c|}
\hline \multirow{2}{*}{$\begin{array}{l}\text { Tumor expression } \\
\text { of antigen }\end{array}$} & \multicolumn{3}{|c|}{ Histotype } & \multirow{2}{*}{$\begin{array}{l}\text { All NSCLC specimens } \\
(n=169)\end{array}$} \\
\hline & Adenocarcinoma $(n=81)$ & Squamous cell carcinoma $(n=88)^{*}$ & p-value & \\
\hline GAGE & $15(18.5 \%)$ & $29(33.0 \%)$ & 0.19 & $44(26.0 \%)$ \\
\hline$>1 \%-\leq 10 \%$ & $5(6.2 \%)$ & $5(5.7 \%)$ & & \\
\hline$>10 \%-\leq 50 \%$ & $2(2.5 \%)$ & $4(4.6 \%)$ & & \\
\hline$>50 \%$ & $8(9.9 \%)$ & $20(22.7 \%)$ & 0.02 & \\
\hline NY-ESO-1 & $8(9.9 \%)$ & $12(13.6 \%)$ & 0.49 & $20(11.8 \%)$ \\
\hline$>1 \%-\leq 10 \%$ & $2(2,5 \%)$ & $1(1.1 \%)$ & & \\
\hline$>10 \%-\leq 50 \%$ & $2(2.5 \%)$ & $1(1.1 \%)$ & & \\
\hline$>50 \%$ & $4(4.9 \%)$ & $10(11.4 \%)$ & 0.13 & \\
\hline SP17 & $4(4.9 \%)$ & $4(4.6 \%)$ & 0.76 & $8(4.7 \%)$ \\
\hline$>1 \%-\leq 10 \%$ & $4(4.9 \%)$ & $4(4.6 \%)$ & & \\
\hline$>10 \%-\leq 50 \%$ & 0 & 0 & & \\
\hline$>50 \%$ & 0 & 0 & & \\
\hline
\end{tabular}

* The survival of the four NOS patients resembled that of patients with squamous cell carcinoma and were therefore included in this group for statistical analysis.

homogenously expressed or clustered. CT antigens have been proposed as markers of cancer stem cells [26], and further studies should be conducted to uncover the identity of this small subset of SP17-positive tumor cells. It is also notable that while the frequency of tumors positive for both GAGE and NY-ESO-1 proteins suggested a degree of coordinated expression of these proteins $(p=0.03)$, neither showed any tendency to co-expression with SP17 $(\mathrm{p}=0.65$ and $\mathrm{p}=1.00$ ) (Figure 2). Unlike GAGE and NY-ESO-1, SP17 is expressed in ciliated normal tissues in addition to testis, indicating that the encoding genes exhibit differences in tissue-specific regulation, which may explain the significant expression dissimilarities observed in NSCLC and other cancers [17]. It further confirms the notion that chromosome $\mathrm{X}$ encoded and autosomal encoded CT antigens exhibit different expression profiles in normal and malignant tissues.

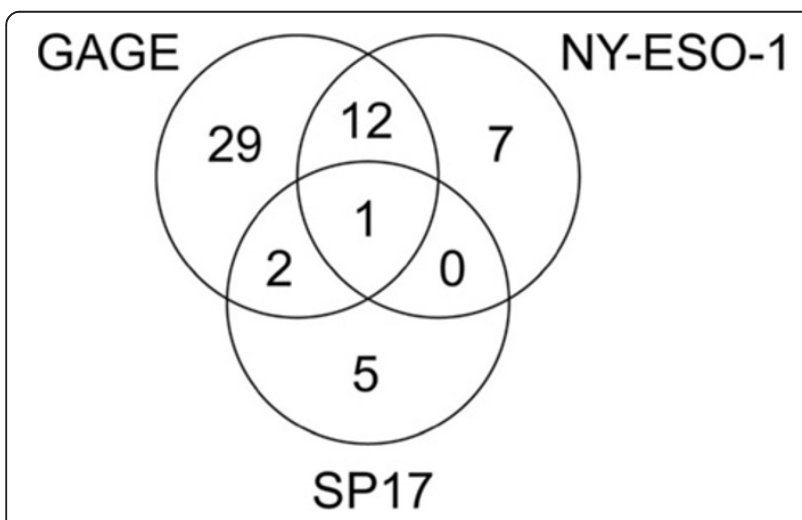

Figure 2 Venn diagram demonstrating overlap between GAGE, NY-ESO-1 and SP17 expression in NSCLC specimens. Numbers represent positive tumors among the total of 169 NSCLC specimens included in the analysis.
The panel of NSCLC included both adenocarcinomas and squamous cell carcinomas. In general, the expression of GAGE, NY-ESO-1 and SP17 CT antigens were not associated with any specific histology type (Table 2), but strongly GAGE-positive tumors ( $>50 \%$ positive tumor cells) were more frequent in squamous cell carcinomas $(p=0.02)$. A similar correlation has been reported between MAGE-A3 and MAGE-A4 and squamous cell carcinoma [20]. Adenocarcinomas and squamous cell carcinomas have been shown to differ in their DNA methylation patterns $[27,28]$, and since promoter hypomethylation is important inducer of $\mathrm{CT}$ antigen gene expression in cancer cells [2], this may explain the differences in CT antigen expression between these two subtypes of NSCLC.

GAGE protein expression significantly correlated with disease progression $(\mathrm{p}=0.02)$, i.e. $17.0 \%(15 / 88)$ of stage I and 35.8\% (29/81) of stage II-IIIa tumors were GAGEpositive (Table 3). NY-ESO-1 expression also tended to associate with advanced disease stages, but not to a statistically significant degree. Similarly, the frequency of MAGE-A4-positive tumors has been reported to be a significantly higher in stage II-IV than stage I NSCLC [20].

The association between CT antigen expression and disease-specific and overall survival was also analyzed for GAGE and NY-ESO-1 (Figure 3); SP-17-positive specimen numbers were too low to allow for a statistical analysis. Although GAGE expression tended to correlate with poor survival, neither GAGE nor NY-ESO-1 was significantly associated with disease-specific or overall survival.

Our results show that the CT antigens GAGE, NY-ESO1 and SP17 are expressed in a considerable proportion of NSCLC and may therefore serve as candidate targets for immunotherapeutic treatments of this disease. Furthermore, GAGE and NY-ESO-1 were present in more 
Table 3 Expression of GAGE, NY-ESO-1 and SP17 in different stages of NSCLC

\begin{tabular}{llll}
\hline Antigen & \multicolumn{3}{c}{ TNM stage } \\
\cline { 2 - 4 } & la-b (n= 88) & II-IIla $(\mathbf{n}=\mathbf{8 1})$ & p-value \\
\hline GAGE & $15(17.0 \%)$ & $29(35.8 \%)$ & 0.02 \\
$>1 \%-\leq 10 \%$ & $4(4.5 \%)$ & $6(7.4 \%)$ & \\
$>10 \%-\leq 50 \%$ & 0 & $6(7.4 \%)$ & \\
$>50 \%$ & $11(12.5 \%)$ & $17(21.0 \%)$ & 0.36 \\
\hline NY-ESO-1 & $9(10.2 \%)$ & $11(13.6 \%)$ & \\
$>1 \%-\leq 10 \%$ & 0 & $3(3.7 \%)$ & \\
$>10 \%-\leq 50 \%$ & $2(2.3 \%)$ & $1(1.2 \%)$ & 0.94 \\
$>50 \%$ & $7(7.9 \%)$ & $7(8.7 \%)$ & \\
\hline SP17 & $4(4.5 \%)$ & $4(4.9 \%)$ & \\
$>1 \%-\leq 10 \%$ & $4(4.5 \%)$ & $4(4.9 \%)$ & \\
$>10 \%-\leq 50 \%$ & 0 & 0 & \\
$>50 \%$ & 0 & 0 & \\
\hline
\end{tabular}

than $50 \%$ of the tumor cells in $63.6 \%(28 / 44)$ and $70 \%$ $(14 / 20)$ of the positive cases, respectively. It seems likely that treatment directed against a tumor antigen broadly expressed within tumors may be most effective, although this has not been demonstrated. The relative homogeneity of GAGE and NY-ESO-1 in NSCLC tumors further strengthens their therapeutic potential, while the scattered expression of SP17 in NSCLC tumors suggests that this is a relatively poor target for NSCLC. Our results demonstrate significant differences in tumor expression of the two chromosome X-encoded CT antigens GAGE, NY-ESO-1 and the autosomal CT antigen SP17 in NSCLC. While only one tumor was positive for all three CT antigens, 56/169 (33.1\%) were positive for at least one of these $\mathrm{CT}$ antigens, demonstrating that immunotherapeutic strategies should aim at different CT antigen targets, including both chromosome X-encoded and autosomal encoded antigens.

\section{Conclusions}

This study determines the expression frequency and correlation with clinical parameters of GAGE, NY-ESO-1 and SP17 CT antigens in NSCLC, which may facilitate

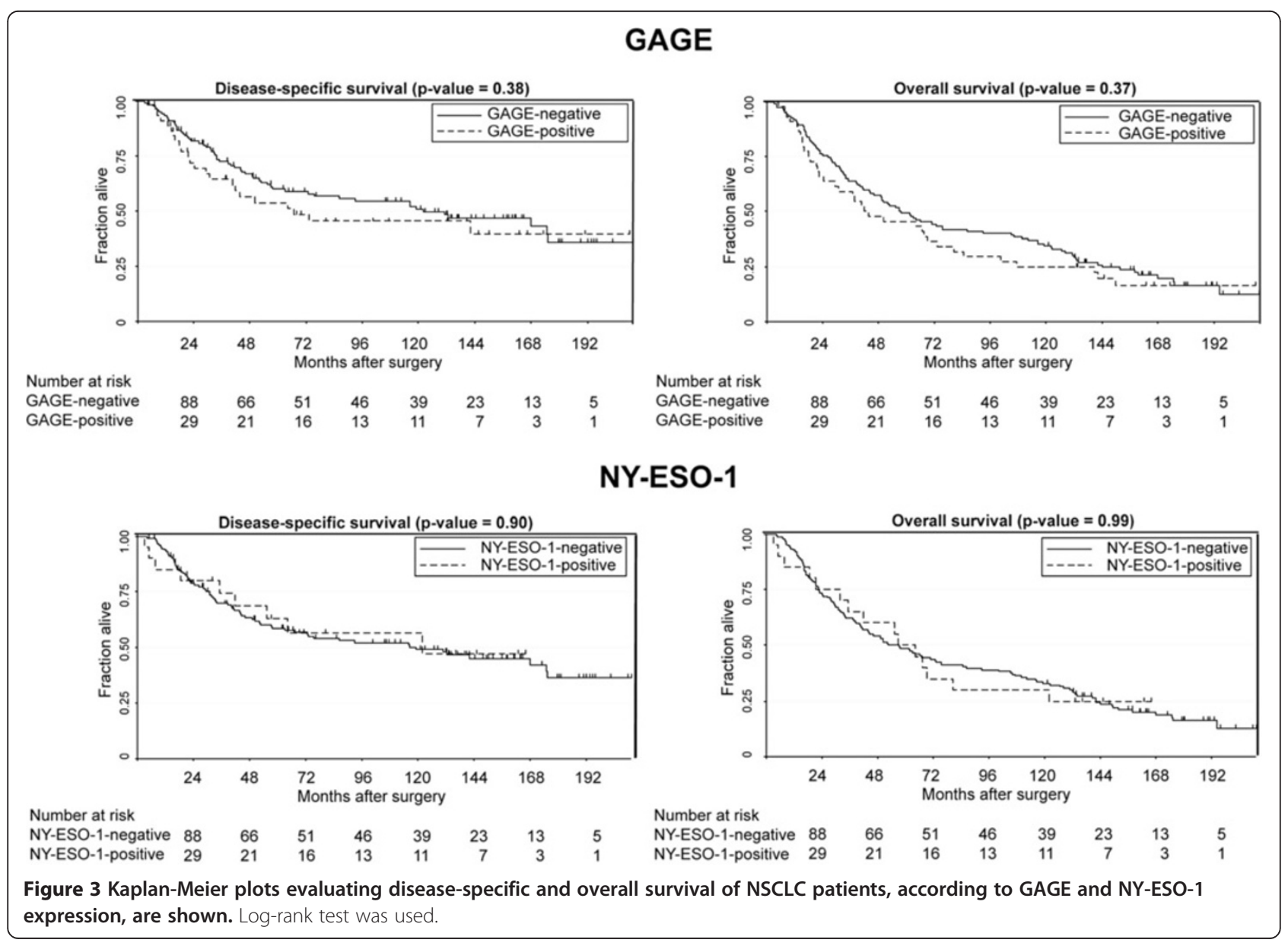


the use of these CT antigens as therapeutic targets for immunotherapy of NSCLC.

\section{Abbreviations \\ CT: Cancer/testis antigen; NSCLC: Non-small cell lung carcinoma.}

\section{Competing interests}

The authors declare that they have no competing interests.

\section{Authors' contributions}

MG designed the experiments, analyzed the data and prepared the manuscript. MP designed the experiments, analyzed the data and prepared the manuscript. KEO designed the experiments and analyzed the data. HJD designed the experiments and prepared the manuscript. All authors read and approved the final manuscript.

\section{Acknowledgements}

We thank Lisbet Mortensen and Ole Nielsen for excellent technical assistance with the immunohistochemical analysis and M. K. Occhipinti-Bender for editorial assistance. This study was supported by the Danish Research Council, the Danish Cancer Society, the Danish Cancer Research Foundation, the Lundbeck Foundation, the LeoPharma Research Foundation, and the Hørslev Foundation.

\section{Author details}

${ }^{1}$ Department of Cancer and Inflammation Research, Institute for Molecular Medicine (IMM), University of Southern Denmark, Winsloewparken 25, 3, DK-5000 Odense C, Denmark. Institute of Clinical Research, University of Southern Denmark, Campusvej 55, DK-5230 Odense, Denmark. ${ }^{3}$ Department of Oncology, Odense University Hospital, Sdr. Boulevard 29, DK-5000 Odense, Denmark. ${ }^{4}$ Department of Pathology, Odense University Hospital, Winsloewparken 15, DK-5000 Odense, Denmark.

Received: 8 April 2013 Accepted: 7 October 2013

Published: 8 October 2013

\section{References}

1. Gjerstorff MF, Burns J, Ditzel HJ: Cancer-germline antigen vaccines and epigenetic enhancers: future strategies for cancer treatment. Expert Opin Biol Ther 2010, 10(7):1061-1075.

2. Simpson AJ, Caballero OL, Jungbluth A, Chen YT, Old L: Cancer/testis antigens, gametogenesis and cancer. Nat Rev Cancer 2005, 5(8):615-625.

3. Caballero OL, Chen YT: Cancer/testis (CT) antigens: potential targets for immunotherapy. Cancer Sci 2009, 100(11):2014-2021.

4. Rao M, Chinnasamy N, Hong JA, Zhang Y, Zhang M, Xi S, Liu F, Marquez VE, Morgan RA, Schrump DS: Inhibition of histone lysine methylation enhances cancer-testis antigen expression in lung cancer cells: implications for adoptive immunotherapy of cancer. Cancer Res 2011, 71(12):4192-4204

5. Kronig H, Hofer K, Conrad H, Guilaume P, Muller J, Schiemann M, Lennerz V, Cosma A, Peschel C, Busch DH, et al: Allorestricted T lymphocytes with a high avidity T-cell receptor towards NY-ESO-1 have potent anti-tumor activity. Int J Cancer 2009, 125(3):649-655.

6. Zhao Y, Zheng Z, Robbins PF, Khong HT, Rosenberg SA, Morgan RA: Primary human lymphocytes transduced with NY-ESO-1 antigen-specific TCR genes recognize and kill diverse human tumor cell lines. J Immunol 2005, 174(7):4415-4423.

7. Molina JR, Yang P, Cassivi SD, Schild SE, Adjei AA: Non-small cell lung cancer: epidemiology, risk factors, treatment, and survivorship. Mayo Clin Proc 2008, 83(5):584-594.

8. Nakagawa K, Noguchi Y, Uenaka A, Sato S, Okumura H, Tanaka M, Shimono $M$, Ali Eldib AM, Ono T, Ohara N, et al: XAGE-1 expression in non-small cell lung cancer and antibody response in patients. Clin Cancer Res 2005, 11(15):5496-5503.

9. Groeper C, Gambazzi F, Zajac P, Bubendorf L, Adamina M, Rosenthal R, Zerkowski HR, Heberer M, Spagnoli GC: Cancer/testis antigen expression and specific cytotoxic T lymphocyte responses in non small cell lung cancer. Int I Cancer 2007, 120(2):337-343.

10. Ohue Y, Eikawa S, Okazaki N, Mizote Y, Isobe M, Uenaka A, Fukuda M, Old L, Oka M, Nakayama E: Spontaneous antibody, and CD4 and CD8 T-cell responses against XAGE-1b (GAGED2a) in non-small cell lung cancer patients. Int J Cancer 2012, 131(5):E649-E658.

11. Nakamura Y, Noguchi Y, Satoh E, Uenaka A, Sato S, Kitazaki T, Kanda T, Soda H, Nakayama E, Kohno S: Spontaneous remission of a non-small cell lung cancer possibly caused by anti-NY-ESO-1 immunity. Lung Cancer 2009, 65(1):119-122.

12. Tsuji T, Altorki NK, Ritter G, Old LJ, Gnjatic S: Characterization of preexisting MAGE-A3-specific CD4+ T cells in cancer patients and healthy individuals and their activation by protein vaccination. J Immunol 2009, 183(7):4800-4808.

13. Chapman CJ, Murray A, McElveen JE, Sahin U, Luxemburger U, Tureci O, Wiewrodt R, Barnes AC, Robertson JF: Autoantibodies in lung cancer: possibilities for early detection and subsequent cure. Thorax 2008, 63(3):228-233.

14. Watanabe $Y$, LePage $S$, Elliott M, Secrist $H$, Tanaka T, Kawahara M, Matsumura A, Hosoe S, Ogawara M, Okada M, et al: Characterization of preexisting humoral immunity specific for two cancer-testis antigens overexpressed at the mRNA level in non-small cell lung cancer. Cancer Immun 2006, 6:3.

15. Shan Q, Lou X, Xiao T, Zhang J, Sun H, Gao Y, Cheng S, Wu L, Xu N, Liu S: A cancer/testis antigen microarray to screen autoantibody biomarkers of non-small cell lung cancer. Cancer Lett 2013, 328(1):160-167.

16. Peled N, Oton AB, Hirsch FR, Bunn P: MAGE A3 antigen-specific cancer immunotherapeutic. Immunother 2009, 1(1):19-25.

17. Gjerstorff MF, Ditzel HJ: Limited SP17 expression within tumors diminishes its therapeutic potential. Tissue Antigens 2012, 80(6):523-527.

18. Gjerstorff MF, Johansen LE, Nielsen O, Kock K, Ditzel HJ: Restriction of GAGE protein expression to subpopulations of cancer cells is independent of genotype and may limit the use of GAGE proteins as targets for cancer immunotherapy. Br J Cancer 2006, 94(12):1864-1873.

19. Gjerstorff MF, Kock K, Nielsen O, Ditzel HJ: MAGE-A1, GAGE and NY-ESO-1 cancer/testis antigen expression during human gonadal development. Hum Reprod 2007, 22(4):953-960.

20. Shigematsu Y, Hanagiri T, Shiota H, Kuroda K, Baba T, Mizukami M, So T, Ichiki Y, Yasuda M, So T, et al: Clinical significance of cancer/testis antigens expression in patients with non-small cell lung cancer. Lung Cancer 2010, 68(1):105-110.

21. Yoshida N, Abe H, Ohkuri T, Wakita D, Sato M, Noguchi D, Miyamoto M, Morikawa T, Kondo S, Ikeda $\mathrm{H}$, et al: Expression of the MAGE-A4 and NY-ESO-1 cancer-testis antigens and T cell infiltration in non-small cell lung carcinoma and their prognostic significance. Int J Oncol 2006, 28(5):1089-1098.

22. Kim SH, Lee S, Lee CH, Lee MK, Kim YD, Shin DH, Choi KU, Kim JY, Park Do Y, Sol MY: Expression of cancer-testis antigens MAGE-A3/6 and NY-ESO-1 in non-small-cell lung carcinomas and their relationship with immune cell infiltration. Lung 2009, 187(6):401-411.

23. Jungbluth AA, Chen YT, Stockert E, Busam KJ, Kolb D, Iversen K, Coplan K, Williamson B, Altorki N, Old LJ: Immunohistochemical analysis of NY-ESO-1 antigen expression in normal and malignant human tissues. Int J Cancer 2001, 92(6):856-860.

24. Schettino C, Bareschino MA, Sacco PC, Maione P, Rossi A, Casaluce F, Sgambato A, Gridelli C: New molecular targets in the treatment of NSCLC. Current Pharma Design 2013, 19(30):5333-5343.

25. Koivunen JP, Mermel C, Zejnullahu K, Murphy C, Lifshits E, Holmes AJ, Choi HG, Kim J, Chiang D, Thomas R, et al: EML4-ALK fusion gene and efficacy of an ALK kinase inhibitor in lung cancer. Clin Cancer Res off J Am Assoc Cancer Res 2008, 14(13):4275-4283.

26. Costa FF, Le Blanc K, Brodin B: Concise review: cancer/testis antigens, stem cells, and cancer. Stem Cells 2007, 25(3):707-711.

27. Carvalho RH, Haberle V, Hou J, van Gent T, Thongjuea S, van ljcken W, Kockx C, Brouwer R, Rijkers E, Sieuwerts A, et al: Genome-wide DNA methylation profiling of non-small cell lung carcinomas. Epigenet Chrom 2012, 5(1):9.

28. Lokk K, Vooder T, Kolde R, Valk K, Vosa U, Roosipuu R, Milani L, Fischer K, Koltsina $\mathrm{M}$, Urgard $\mathrm{E}$, et al: Methylation markers of early-stage non-small cell lung cancer. PLoS One 2012, 7(6):e39813.

doi:10.1186/1471-2407-13-466

Cite this article as: Gjerstorff et al: Analysis of GAGE, NY-ESO-1 and SP17 cancer/testis antigen expression in early stage non-small cell lung carcinoma. BMC Cancer 2013 13:466. 